\title{
Multi-narrowband signals receiving method based on analog-to-information convertor and block sparsity
}

\author{
Hongyi $\mathrm{Xu}^{1}$, Haiqing Jiang ${ }^{2, *}$, and Chaozhu Zhang ${ }^{1}$ \\ 1. School of Information and Communication Engineering, Harbin Engineering University, Harbin 150001, China; \\ 2. School of Information and Electronic Engineering, Beijing Institute of Technology, Beijing 100081, China
}

\begin{abstract}
The analog-to-information convertor (AIC) is a successful practice of compressive sensing (CS) theory in the analog signal acquisition. This paper presents a multi-narrowband signals sampling and reconstruction model based on AIC and block sparsity. To overcome the practical problems, the block sparsity is divided into uniform block and non-uniform block situations, and the block restricted isometry property and sub-sampling limit in different situations are analyzed respectively in detail. Theoretical analysis proves that using the block sparsity in AIC can reduce the restricted isometric constant, increase the reconstruction probability and reduce the sub-sampling rate. Simulation results show that the proposed model can complete sub-sampling and reconstruction for multi-narrowband signals. This paper extends the application range of AIC from the finite information rate signal to the multi-narrowband signals by using the potential relevance of support sets. The proposed receiving model has low complexity and is easy to implement, which can promote the application of CS theory in the radar receiver to reduce the burden of analog-todigital convertor (ADC) and solve bandwidth limitations of ADC.
\end{abstract}

Keywords: compressive sensing (CS), block sparsity, analog-toinformation convertor (AIC), multi-narrowband signals.

DOI: $10.21629 /$ JSEE.2017.04.03

\section{Introduction}

The compressive sensing (CS) theory was proposed by Donoho et al. [1 -3] in 2006. It can obtain a non-adaptive linear projection at a rate far below the Nyquist frequency form signal and reconstruct it in a high probability through algorithms using the prior knowledge of sparsity.

CS technology has been applied to many research fields, including radar signal processing, wireless sensor networks, image acquisition, medical signal processing, etc. These applications are usually built on the basis of digital signal processing, mainly used in the finite-dimensional

\footnotetext{
Manuscript received November 08, 2016.

*Corresponding author.

This work was supported by the National Natural Science Foundation of China (61172159).
}

digital signal. In the analog domain, Tropp, et al. studied the feasibility of applications of CS in analog information collection [4-7]. Baraniuk, a research team from Rice University, designed an analog-to-information convertor (AIC) based on CS theory [8-12]. After that, Candès of California Institute of Technology developed a compression sampling chip named random modulation pre-integration (RMPI) on the basis of AIC [13]. Daponte et al. studied the effects of the jitter in the pseudo random binary sequence used in the mixing step of the AIC [14]. As the most successful application of CS theory in the analog domain, the AIC sampling model has been widely used in radar, communications, cognitive radio and other fields [15-19]. In 2010, Mishali et al. improved the framework of AIC and presented a modulated wideband conversion (MWC) [20-22]. However, it has high computational complexity and strict requirements for the hardware with parallel structure.

In terms of reconstruction, various algorithms can be used to reconstruct the original signal using compressive samples [23-27]. Based on the framework of traditional algorithms, Lee et al. proposed a novel support detection method which was referred to maximize the posteriori support detection [28]. Recent studies have addressed reconstruction algorithms specially for the original signal with special structure characteristics and the advantages of block sparsity are re-emphasized by scholars. By analyzing the characteristic of block sparse signals, Eldar et al. developed a block-MP and block-BOMP algorithm on the basis of matching pursuit (MP) and orthogonal MP (OMP) $[29,30]$. Shekaramiz et al. investigated the recovery of block-sparse signals via multiple measurement vectors in the presence of noise [31]. Shah et al. explored the block 11-norm regularizers for the compressive image recovery, which used the potential correlation between adjacent pixels [32].

In reality, many of the original signals, such as the multiband narrowband signal that the radar receiver may en- 
counter, have block sparse structures. By promoting the application to receiver, the sampling rate and the sampling burden of the analog-to-digital convertor (ADC) will be greatly reduced. Meanwhile, the problem of bandwidth limitations on ADC can also be solved.

\section{Digital CS theory}

Different from the traditional Nyquist theory, the CS theory suggests that as long as the signal is compressible or sparse in a transform domain, an observation matrix incoherent with the transform bases can be used to project the resulting high-dimensional signal onto a lower dimensional space, and then the original signal can be reconstructed in a high probability from the few projections by solving an optimization problem. It has been proved that such projections contain enough information for reconstructing signals. In this framework, the sampling rate is not determined by the bandwidth of the signal, but depends on the structure and content of the information in the signal.

The signal $\boldsymbol{x} \in \mathbf{R}^{N \times 1}$ to be processed is the one-dimensional real-valued time series with element $x[n](n=1,2, \ldots, N)$. A signal is called the time-domain sparse signal if most elements of $\boldsymbol{x}$ are zero or approximate to zero. However, most of the signals in the nature are nonsparse in the time domain but sparse in some transform domain. Such signals are called the compressible signal. Assume a set of orthonormal basis $\boldsymbol{\Psi}=\left[\psi_{1}, \psi_{2}, \ldots, \psi_{N}\right]$ in the space $\mathbf{R}^{N}$, then there are

$$
\boldsymbol{x}=\sum_{i=1}^{N} s_{i} \psi_{i}=\boldsymbol{\Psi}^{\mathrm{T}} \boldsymbol{s}
$$

where $s=\left\{s_{i}\right\}_{i=1}^{N}$ is a column vector with an $N \times 1$ dimension, and $s_{i}=\left\langle\boldsymbol{x}, \psi_{i}\right\rangle=\psi_{i}^{\mathrm{T}} \boldsymbol{x}$. If the number of support set in $s_{i}=\left\langle\boldsymbol{x}, \psi_{i}\right\rangle$ is not greater than $k$, the signal can be called a $k$-sparse signal.

With the observation matrix $\boldsymbol{\Phi} \in \mathbf{R}^{M \times N}(M<<N)$, the linear measurements $\boldsymbol{y} \in \mathbf{R}^{M}$ of $\boldsymbol{x}$ can be expressed as

$$
\boldsymbol{y}=\boldsymbol{\Phi} \boldsymbol{x}=\boldsymbol{\Phi} \boldsymbol{\Psi}^{\mathrm{T}} \boldsymbol{s} .
$$

In the process of reconstruction, the original values of $\boldsymbol{x}$ need to be obtained according to (2). Because the dimension of $\boldsymbol{y}$ is much smaller than $\boldsymbol{x}$, (2) can have infinite solutions. If the original signal is $k$-sparse with $\boldsymbol{\Psi}$, the sparse expression $s$ can be reconstructed algorithmically, thereby we can obtain the original signal. In order to facilitate the reconstruction process, $\Theta=\boldsymbol{\Phi} \boldsymbol{\Psi}^{\mathrm{T}}$ is defined as a recovery matrix.

Definition 1 Let $\Theta$ be a given matrix. If there exists a restricted isometric constant (RIC) $\delta_{k}$ satisfying

$$
\left(1-\delta_{k}\right)\|s\|_{\ell_{2}}^{2} \leqslant\|\Theta s\|_{\ell_{2}}^{2} \leqslant\left(1+\delta_{k}\right)\|s\|_{\ell_{2}}^{2}
$$

then $\Theta$ satisfies the restricted isometry property (RIP). And $s$ has a unique solution if $\delta_{K} \in(0,1)$.

For the given observation matrix, whether it meets the requirement of RIP is extremely difficult to verify. Baraniuk et al. [33] proposed an equivalent conditions of RIP that the observation matrix is irrelevant with the sparsity base matrix. The correlation coefficience is defined as below:

$$
\mu(\boldsymbol{\Phi}, \boldsymbol{\Psi})=\sqrt{N} \max _{1 \leqslant i, j \leqslant N}\left|\left\langle\varphi_{i}, \psi_{j}\right\rangle\right|
$$

where $\mu \in[1, \sqrt{N}]$ defines the maximum correlation of column vectors. The smaller the correlation coefficient is, the more original information retained in measurements and the higher the probability of accurate reconstruction can be gotten. Most random matrices in uniform distribution are able to meet RIP in the greater probability. Common observation matrices include the Gaussian random matrix, the Bernoulli random matrix, the part Fourier matrix, the part Hadamard matrix, the sparsity random matrix, etc.

The reconstruction algorithms can be grouped into the following two categories:

\section{(i) Convex optimization algorithm}

These methods obtain approximate solutions of signals by solving a non-convex problem into a convex problem such as basis pursuit, interior-point method, projected gradient method, and iterative thresholding.

(ii) Greedy algorithm

Greedy algorithms iteratively approximate the coefficients and the support of the original signal. They have the advantage of fast convergency and are easy to implement. Commonly used greedy algorithms include MP, OMP, stagewise OMP (StOMP), regularized OMP (ROMP) and compressive sampling MP (CoSaMP). These algorithms require more samples than $\mathrm{BP}$ in exchange for reducing the computational complexity.

\section{CS in analog domain}

The original CS theory was proposed to solve the problem of high sampling rate. CS technology has a sound performance in digital domain with discrete sequence. In order to reduce the pressure of sampling device, promoting the CS technology to the analog domain is considered the key which can subvert the traditional sampling mode. How to achieve the CS physically for the continuous-time signal is the main research content of CS in the analog domain. In other words, it is a procedure completing the compression and recovery of information on the time domain. Currently, $\mathrm{AIC}$ is a main method of CS in the analog domain. 


\subsection{AIC sampling model}

Physical implementation structure of the AIC is shown in Fig. 1, which includes the mixer for pre-modulation, the low pass filter for integral and low-speed ADC.

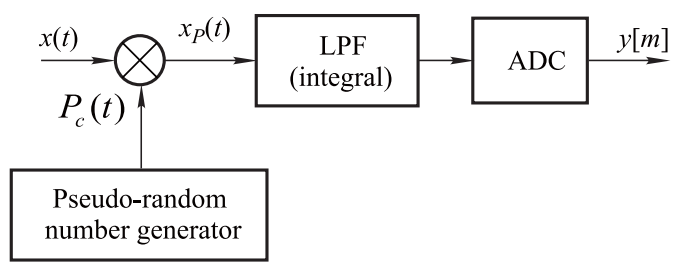

Fig. 1 AIC physical implementation structure

First, the analog signal $x(t)$ needs to be modulated with a high rate random wave $P_{c}(t)$ which is a pseudo-random sequence with \pm 1 in this paper and has a frequency greater than or equal to the Nyquist sampling rate of the analog signal $x(t)$. Second, the modulated waveform $x_{P}(t)$ enters a low pass filter (LPF) to compress information, which is a process of integration. Finally, the CS sampling data can be obtained through the traditional low-rate ADC sampling.

The pseudo-random sequence $P_{c}(t)$ changes with the frequency of $W$, and each change randomly takes 1 or -1 . The mathematical expression is shown as follows:

$$
P_{c}(t)=\varepsilon_{n}, \quad t \in\left[\frac{n}{W}, \frac{n+1}{W}\right] ; n=0,1, \ldots, W-1
$$

where $\varepsilon_{n}$ takes the value of \pm 1 .

Assume the input analog signal has a limited information rate, namely, the signal can be represented with $k$ parameters in a set of continuous bases in each unit of time. More specifically, the analog signal is made up of a discrete and limited number of weighting dictionary components or continuous bases composition. Now define a set of orthonormal basis $\boldsymbol{\Psi}=\left[\psi_{1}, \psi_{2}, \ldots, \psi_{N}\right]$ in the space $\mathbf{R}^{N}$, then

$$
x(t)=\sum_{n=1}^{N} \alpha_{n} \psi_{n}(t) .
$$

When $\alpha_{n}$ has few non-zero coefficients, the input $x(t)$ can be considered a sparse signal. Although each dictionary component or orthogonal base may have a relatively wide bandwidth, the signal itself has only a few degrees of freedom. When the observation matrix is $\Phi \in$ $\mathbf{R}^{M \times N}(M \ll N)$, the linear measurements $\boldsymbol{y} \in \mathbf{R}^{M}$ can be expressed as

$$
\boldsymbol{y}=\boldsymbol{\Phi} \boldsymbol{x}=\boldsymbol{\Phi} \boldsymbol{\Psi}^{\mathrm{T}} \boldsymbol{s} .
$$

Distinct from the traditional discrete CS reconstruction process, the AIC faces continuous-time signals directly in the analog domain. Hence, there is no observation matrix in this system during the reconstruction process. To predigest the reconstruction process, the recovery matrix of AIC system $\Theta=\boldsymbol{\Phi} \boldsymbol{\Psi}^{\mathrm{T}}$ is defined as in Section 2. After the process of random demodulation and low-pass filter, the low-speed sampling result $y[m]$ is generated, which can be described as

$$
y[m]=\sum_{n=1}^{N} \alpha_{n} \int_{-\infty}^{\infty} \psi_{n}(\tau) p_{c}(\tau) h(m M-\tau) \mathrm{d} \tau .
$$

The recovery matrix $\Theta$ can be written as

$$
\theta_{m, n}=\int_{-\infty}^{\infty} \psi_{n}(\tau) p_{c}(\tau) h(m M-\tau) \mathrm{d} \tau .
$$

In order to facilitate the simulation, the LPF in (9) is replaced by an ideal integrator as

$$
\begin{aligned}
& y[m]=\int_{m T_{e}}^{(m+1) T_{e}} p_{c}(t) x(t) \mathrm{d} t= \\
& \sum_{n=m \cdot N / M}^{(m+1) N / M} p_{c}[n] \int_{m T_{e}}^{(m+1) T_{e}} x(t) \mathrm{d} t
\end{aligned}
$$

where $T_{e}$ is the equivalent sampling interval. If AIC acquires the signal in the period of $T, M T=N T_{e}$. Then the analog signal $x(t)$ is represented as a sequence of Nyquist sampling, and (10) can be rewritten as

$$
y[m]=\sum_{n=m N / M}^{(m+1) N / M} p_{c}[n] \cdot x[n] .
$$

It can be expressed as a matrix-vector form:

$$
\boldsymbol{y}=\boldsymbol{D P} \boldsymbol{x}
$$

where $\boldsymbol{y}=\left[y_{0}, y_{1}, \ldots, y_{M-1}\right]^{\mathrm{T}}$ are measurements of AIC, $\boldsymbol{x}=\left[x_{0}, x_{1}, \ldots, x_{N-1}\right]^{\mathrm{T}}$ is a Nyquist sampling sequence of the original analog signal, $\boldsymbol{P}$ is the random sequence in the form of a unit matrix

$$
\boldsymbol{P}=\left[\begin{array}{llll}
p_{c}[0] & & & \\
& p_{c}[1] & & \\
& & \ddots & \\
& & & p_{c}[N-1]
\end{array}\right] .
$$

In the case of a discrete sequence, the integrator aims at accumulating $N / M$ sampling values, thereby achieving the purpose of compressing observation signals. For instance, when $N=9, M=4, \boldsymbol{D}$ can be expressed as

$$
\boldsymbol{D}=\left[\begin{array}{llllllllll}
1 & 1 & \frac{1}{4} & & & & & & \\
& & \frac{3}{4} & 1 & \frac{2}{4} & & & & \\
& & & & \frac{2}{4} & 1 & \frac{3}{4} & & \\
& & & & & & & & \\
& & & & & & \frac{1}{4} & 1 & 1
\end{array}\right] .
$$


Thus the recovery matrix $\Theta$ can be found as $\Theta=$ $D P \Psi$ in the AIC sampling model in the form of a matrixvector.

Signal reconstruction algorithms can be grouped into convex optimization algorithms and greedy algorithms as described in Section 2. Compared with convex optimization algorithms, greedy algorithms are easier for engineering implementation because of their lower calculation load. Considering the high demand of computing speed for signal reconstruction, the AIC system using greedy algorithms would have a better performance.

\subsection{Simulation and analysis of AIC sampling model}

Discrete Fourier transform (DFT) bases are selected as orthogonal bases. The original input signal contains three frequency components: $100 \mathrm{~Hz}, 300 \mathrm{~Hz}$ and $350 \mathrm{~Hz}$. The Nyquist sampling rate $f_{N y q}=700 \mathrm{~Hz}$, the simulation sampling frequency $f_{s}=800 \mathrm{~Hz}$, the signal length $N=256$, the compression sampling number $M=40$, the subNyquist sampling rate $Q=0.179$. The OMP is used in this simulation. Reconstruction results are shown in Fig. 2.

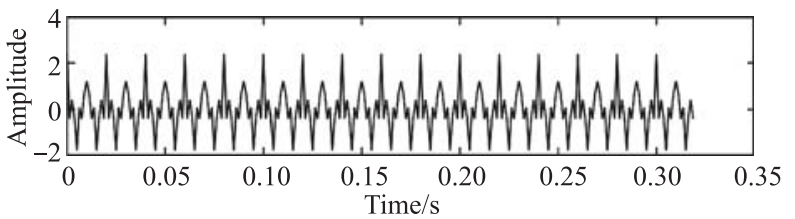

(a) Original signal $(K=6, M=40, Q=0.17857)$

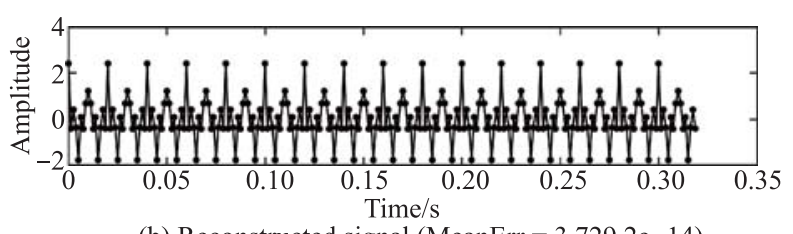

(b) Reconstructed signal (MeanErr $=3.7292 \mathrm{e}-14)$

Fig. 2 Results of reconstruction by AIC

The simulation results show that average reconstruction errors are in the magnitude of $10^{-14}$ or less when the subsampling rate is $Q=0.179$ in the time domain and frequency domain. It can be considered that the reconstructed signal contains almost complete information of the original signal. As expected, the AIC frame can realize the CS procedure for limited information rate signals in the analog domain and most information can be recovered effectively by using greedy algorithms.

In general, the traditional AIC sampling model has the following advantages:

(i) It can obtain information efficiently and reconstruct the original signal accurately in a rate far below the Nyquist sampling rate.

(ii) The whole process completes compression and sensing simultaneously, in other words, it can acquire signals in low-speed and digitize the input signal at the same time. (iii) On account of its simple structure, the AIC sampling model performs better with its universality and implementation.

Nevertheless, coupled with traditional greedy algorithms, the AIC sampling model also has some disadvantages. A major problem is that AIC can be only applied efficaciously to limit the information rate signal. It means that the analog input signal must be made up of discrete and limited weighted consecutive bases or dictionary components.

As is shown in Fig. 3, when the AIC sampling model is applied for narrowband signals or certain non-strict sparse signals which contain continuous spectrum, traditional greedy algorithms like OMP will have serious mismatch in the choice of orthogonal basis. This problem is due to the single atom searching principle in the step of calculating the correlation. On the other hand, the continuous spectrum will greatly increase the amount of calculation so that the receiver cannot achieve the real-time reception. This issue will be resolved in the next section.

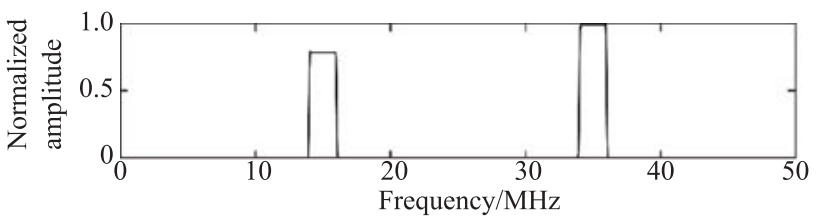

(a) Frequency spectrum of original signal $(K=168, M=400, Q=0.27)$

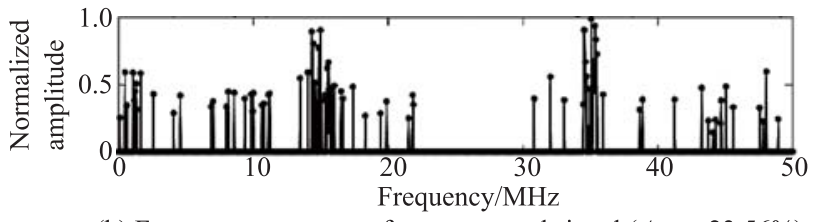

(b) Frequency spectrum of reconstructed signal $(A c c=23.56 \%)$

Fig. 3 Mismatch on reconstruction by OMP

\section{CS model of block sparse signals}

\subsection{Block sparse signal}

Assume that the signal $\boldsymbol{x} \in \mathbf{R}^{N}$ is composed of several blocks with length $d_{l}(l \geqslant 1)$ respectively. Block sparse signals are defined as

$$
\boldsymbol{x}=[\underbrace{x_{1}, \ldots, x_{d_{1}}}_{\boldsymbol{x}^{\mathrm{T}}[1]}, \ldots, \underbrace{x_{N-d_{L}+1}, \ldots, x_{N}}_{\boldsymbol{x}^{\mathrm{T}}[L]}]
$$

where $\boldsymbol{x}[l]$ denotes the $l$ th sub-blocks of signal $\boldsymbol{x}$ with length $d_{l}$.

Compared with the typical $k$-sparse signal which has at most $K$ nonzero coefficients randomly, non-zero coefficients of the block sparse signal distributed only in certain blocks. A block $K$-sparse signal $\boldsymbol{x}$ is defined as a vector 
that satisfies $\|\boldsymbol{x}\|_{2,0} \leqslant K$, where $\|\boldsymbol{x}\|_{2,0}$ is described as

$$
\|\boldsymbol{x}\|_{2,0}=\sum_{t=1}^{L} \xi\left(\boldsymbol{x}\|t\|_{2}\right)
$$

where $\xi$ is an indicator function, $\xi\left(\boldsymbol{x}\|t\|_{2}>0\right)=1$ and 0 otherwise. Moreover, when $d_{1}=d_{2}=\cdots=d_{L}=d$, the signal is considered into the uniform block $K$-sparse. In contrast, it is called as the non-uniform block $K$-sparse when it has different lengths of each block. When $d=1$, block-sparsity retrogrades to the conventional sparsity in which non-zero values are randomly distributed. In this paper, unless otherwise specified, the block $K$-sparse refers to the uniform block $K$-sparse. A uniform block sparse signal can be expressed as

$$
\boldsymbol{x}=[\underbrace{x_{1}, \ldots, x_{d}}_{\boldsymbol{x}^{\mathrm{T}}[1]}, \underbrace{x_{d+1}, \ldots, x_{2 d}}_{\boldsymbol{x}^{\mathrm{T}}[2]}, \ldots, \underbrace{x_{N-d+1}, \ldots, x_{N}}_{\boldsymbol{x}^{\mathrm{T}}[L]}] .
$$

Many practical signals or problems have the form of block spars, such as multi-measurement vector problems, multi-band narrowband signals, and radar pulse signals. Therefore, reconstruction algorithms of these sparse signals with certain specific structure need to be studied furtherly.

\subsection{CS model of block sparse signals}

The process of CS with the block sparse signal is shown as follows:

$$
\boldsymbol{y}=\boldsymbol{\Phi} \boldsymbol{x}_{a}=\boldsymbol{\Phi} \boldsymbol{\Psi}^{\mathrm{T}} \boldsymbol{x}=\boldsymbol{\Theta} \boldsymbol{x}
$$

where $\boldsymbol{x}_{a}$ is the original signal, $\boldsymbol{\Psi}$ is the orthonormal base, $\boldsymbol{x}$ is the block sparsity projection of $\boldsymbol{x}_{a}$ in $\boldsymbol{\Psi}, \boldsymbol{y}$ is the measurement vector. The observation matrix $\boldsymbol{\Phi} \in$ $\mathbf{R}^{M \times N}(M<N)$ is actually a process of sampling in $\mathrm{CS}$. The recovery matrix $\Theta=\boldsymbol{\Phi} \boldsymbol{\Psi}^{\mathrm{T}}$ defined in Section 2 can be partitioned into the block distribution $I=$ $\left\{d_{1}, \cdots, d_{L}\right\}$, similar to the signal

$$
\boldsymbol{\Theta}=[\underbrace{\theta_{1}, \ldots, \theta_{d_{1}}}_{\boldsymbol{\Theta}[1]}, \ldots, \underbrace{\theta_{N-d_{L}+1}, \ldots, \theta_{N}}_{\boldsymbol{\Theta}[L]}]
$$

where $\boldsymbol{\Theta}[i](i=1,2, \ldots, L)$ is a sub-measurement matrix of $M \times d$. Then (18) can be re-expressed by (15) and (19) as

$$
\begin{gathered}
\boldsymbol{y}=(\theta[1] \quad \theta[2] \ldots \theta[q])\left(\boldsymbol{x}^{\mathrm{T}}[1] \quad \boldsymbol{x}^{\mathrm{T}}[2] \ldots \boldsymbol{x}^{\mathrm{T}}[q]\right)^{\mathrm{T}}= \\
\sum_{i=1}^{q} \theta[i] x[i] .
\end{gathered}
$$

It can bring many benefits rewriting the $\mathrm{CS}$ process to the form of block when the signal is known as the block sparse signal. In the aspect of signal reconstruction, the feature that non-zero values appear concentrated makes the recovery matrix easier to meet the RIP in a block form. In the aspect of sub-sampling, the prior knowledge of signal structure form block sparsity can further reduce the required number of sub-samples under the premise of guaranteeing the reconstruction probability, namely, reduce the sub-sampling rate. These two corollaries will be demonstrated in detail in the following sections.

\subsubsection{Block-RIP and RIC}

To ensure that the sparsity expression $\boldsymbol{x}$ of the original signal $\boldsymbol{x}_{a}$ can be reconstructed stably from the measured values $\boldsymbol{y}$ in the conventional sparsity concept, the recovery matrix $\Theta$ needs to meet RIP. In other words, there exists a $\delta_{K} \in(0,1)$ satisfying (3). Reference [34] generalized the RIP to the block-sparsity setting and proposed the block restricted isometry property (Block-RIP).

Definition 2 Let $\Theta$ be a given matrix. Then $\Theta$ satisfies the block RIP over $I=\left\{d_{1}, \ldots, d_{L}\right\}$ with the parameter $\delta_{K \mid I}$ if for every $\boldsymbol{x} \in \mathbf{R}^{N}$ it is block $K$-sparse over $I$, thus we have

$$
\left(1-\delta_{K \mid I}\right)\|\boldsymbol{x}\|_{2}^{2} \leqslant\|\boldsymbol{\Theta} \cdot \boldsymbol{x}\|_{2}^{2} \leqslant\left(1+\delta_{K \mid I}\right)\|\boldsymbol{x}\|_{2}^{2}
$$

where $\delta_{K \mid I}$ is the restricted isometric constant (RIC) of the block $K$-sparse $\boldsymbol{x}$ over $I$. From [28], we know if $\boldsymbol{\Theta}$ satisfies the block-RIP with $\delta_{2 K \mid I}<1$, there is a unique value of $\boldsymbol{x}$ consistent with (18). And the smaller RIC means that the signal can be reconstructed at a higher probability.

Corollary 1 Suppose a signal is block $K$-sparse over $I$ and $k$-sparse with $k=K d$, then RIC satisfies $\delta_{K \mid I} \leqslant \delta_{k}$.

Proof Refering to the perspective of the union of subspaces [35], the arbitrary $k$-sparse signal $\boldsymbol{x} \in \mathbf{R}^{N}$ can be seen as a union of the finite-dimensional linear subspaces $\mathcal{A}_{r}$.

$$
\mathcal{A}_{r}=\bigcup_{j=1}^{r} \mathcal{S}_{j}
$$

where $\mathcal{S}_{j}$ is the subspace with $k$ dimension in $\mathbf{R}^{N}, \mathcal{A}_{r}$ is the union of $r$ subspaces, $\delta_{k}^{A_{r}}$ is RIC of $\boldsymbol{x} \in \mathcal{A}_{r}$ which meets (3), $r$ represents all cases of possible distribution of non-zero values in the sparse signal. Obviously, the fewer $r$ is, the easier the observation matrix can meet RIP, so the less $\delta_{k}^{A_{r}}$ we have. Thus, it can be derived that

$$
0<\delta_{k}^{A_{1}}<\delta_{k}^{A_{2}}<\cdots<\delta_{k}<1 .
$$

Now suppose that a signal is block $K$-sparse, then $r=$ $\left(\begin{array}{l}L \\ K\end{array}\right)$. When it is considered to be a random sparse signal with $k=K d$ non-zero values, $r^{\prime}=\left(\begin{array}{l}N \\ k\end{array}\right)$. It can be 
calculated that $r<r^{\prime}$, the number of distribution situations in block sparsity is less than the conventional random sparsity.

In the process of CS and reconstruction in AIC, adopting the concept of the block sparsity provides a smaller RIC so that the original signal can be reconstructed at a higher probability.

\subsubsection{Sub-Nyquist sampling limit}

In fact, the sub-sampling rate has a lower bound, which depends on the sparsity level of the original signal. When the prior knowledge of block sparsity is known, the performance of sub-Nyquist sampling can be further enhanced. In this section, we still use the perspective of the union of subspaces that the arbitrary $k$-sparse signal $\boldsymbol{x} \in \mathbf{R}^{N}$ can be seen as a union of the finite-dimensional linear subspaces $\mathcal{A}$.

$$
\mathcal{A}=\bigcup_{j}^{m_{k}} \mathcal{S}_{j}
$$

where $\mathcal{S}_{j}$ is the subspace with $k$ dimensions in $\mathbf{R}^{N}, \mathcal{A}$ is the union of $m_{K}$ subspace. For a uniform block $K$-sparse signal, $m_{K}=\left(\begin{array}{l}L \\ K\end{array}\right)$, where $L$ is the total number of blocks in (18). And $m_{k}=\left(\begin{array}{c}N \\ k\end{array}\right)$ when the same signal is regarded as the common random $k$-sparse signal with $k=K d$. Combining conclusions in [29] with the block sparsity, we have

Theorem 1 Let $\boldsymbol{\Theta} \in \mathbf{R}^{M \times N}(M<<N)$ be a recovery matrix. $\boldsymbol{x} \in \mathbf{R}^{N}$ is the uniform block $K$-sparse signal over $I=\left\{d_{1}=\cdots=d_{L}=d\right\}$. For any $t>0$, let

$$
M \geqslant \frac{2}{c \delta}\left(\ln 2 m_{K}+k \ln \frac{12}{\delta}+t\right)
$$

then $\Theta$ satisfies the block-RIP (21) of $\delta_{K \mid I}=\delta$ with the probability at least $1-\mathrm{e}^{t}$. The constant $c>0$ only depends on the distribution of the entries in $\Theta$ and $c=7 / 18$ if the entries of $\Theta$ are independent identically distributed (i.i.d.) normal.

Corollary 2 The uniform block sparsity model has a less low limit of number of measurements than that of the conventional random sparsity model in CS.

Proof From the perspective of the approximation, $\ln 2 m_{k}$ is the dominant factor in (25). Hence, there exists a proportional relationship between $m_{k}$ and the lower limit of $M$. Apparently, the random sparsity model takes all combinations of the non-zero value distribution into account, while the uniform block sparsity model only considers the case that non-zero values locate into block according to $I=\left\{d_{1}=\cdots=d_{L}=d\right\}$. Then we have $m_{K}<m_{k}$.

Corollary 3 The low limit of measurements number in the non-uniform block sparsity model is higher than the situation of the uniform block sparsity model segmented with the maximum block length, but is not higher than the situation segmented with the minimum block length.

Proof For the non-uniform block sparse signal, it can be segmented into two extreme forms as Fig. 4 shows.

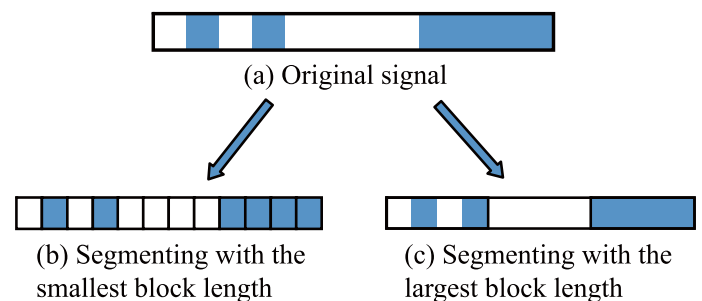

Fig. 4 Segmenting of the original signal

Segment the original signal (a) with a minimum block length to structure a new uniform block sparse signal (b). In this way, big non-zero blocks are cut into several contiguous small blocks. The increase of the number of nonzero blocks and total number of blocks make $m_{K(b)}>$ $m_{K(a)}$. An extreme example is that the minimum block length is one, then (b) degrades into a conventional random sparse signal after segmenting with $m_{k} \geqslant m_{K(b)}>$ $m_{K(a)}$.

Then segment the original signal (a) with a maximum block length to structure a new uniform block sparse signal (c). In this case, numbers of non-zero blocks $K_{(a)} \geqslant K_{(c)}$, and total numbers of blocks satisfy $L_{(a)} \geqslant L_{(c)}$, thereby $m_{K(a)} \geqslant m_{K(c)}$.

Combining both segmenting cases, we have $m_{k} \geqslant$ $m_{K(b)}>m_{K(a)}>m_{K(c)}$.

\section{Reconstruction method for narrowband signal based on block sparsity}

\subsection{Block sparsity reconstruction algorithm}

In the process of reconstruction, common reconstruction algorithms usually ignore the certain inherent structure that the practical signals generally have. For the recovery matrix $\Theta \in \mathbf{R}^{M \times N}(M<N)$ and the measurement vector $\boldsymbol{y} \in \mathbf{R}^{M}$, the model of block sparse signal reconstruction can be written as

$$
\begin{gathered}
\min _{\boldsymbol{x}}\|\boldsymbol{x}\|_{2,0} \\
\text { s.t. } \boldsymbol{\Theta} \boldsymbol{x}=\boldsymbol{y} .
\end{gathered}
$$

The function above is also an NP problem similar to the model of the general sparse signal reconstruction. And it can be transformed into

$$
\begin{gathered}
\min _{\boldsymbol{x}}\|\boldsymbol{x}\|_{2,1} \\
\text { s.t. } \boldsymbol{\Theta} \boldsymbol{x}=\boldsymbol{y} .
\end{gathered}
$$

Equation (27) is a mixed-norm minimization problem where the L-OPT algorithm is commonly used. Nevertheless, the L-OPT algorithm is not straightforward for strong 
real-time characteristic in radar reconnaissance receiver in consideration of its high computational complexity. For this reason, this paper uses greedy algorithms for signal reconstruction. On the basis of MP and OMP algorithms, [26] proposed BMP and BOMP algorithms considering the concept of block sparsity. Different from BMP, BOMP coupled with the idea of the orthogonalization can satisfactorily avoid the repetitive match, and perform a higher reconstruction accuracy. Moreover, it can also satisfy the requirement of real-time property in a small amount of calculation. At this point, this paper uses BOMP to reconstruct signals.

Procedure of BOMP is summarized as follows:

Step 1 Initialization. Initialize residuals $\boldsymbol{r}_{0}=\boldsymbol{y}$.

Step 2 Block selection. Select the best matching block compared with residuals $r_{t-1}$ in the $t$ th iteration.

$$
i_{t}=\arg \max \left\|\boldsymbol{\Phi}^{\mathrm{H}}[i] \boldsymbol{r}_{t-1}\right\|_{2} \text {. }
$$

Step 3 Once the index $i_{t}$ is chosen, compute $\boldsymbol{x}[i]$ and find optimal coefficients by

$$
\min \left\|\boldsymbol{y}-\sum_{i \in I} \boldsymbol{\phi}[i] \boldsymbol{x}[i]\right\|_{2} .
$$

Step 4 Update the support block and residuals

$$
\begin{gathered}
I_{t}=I_{t-1} \cup i_{t} \\
\boldsymbol{r}_{t}=\boldsymbol{y}-\sum_{i \in I} \boldsymbol{\phi}[i]\left(\phi^{\dagger}[i] \boldsymbol{y}\right)
\end{gathered}
$$

where $\phi^{\dagger}[i]=\left[\boldsymbol{\phi}^{\mathrm{T}} \boldsymbol{\phi}\right]^{-1} \boldsymbol{\phi}^{\mathrm{T}}$ is the pseudoinverse of $\boldsymbol{\phi}[i]$.

In BOMP, the inner product is computed by block, and in each iteration, the block with the largest correlation to the residual is added to the support set. Then the residual is updated for the next loop. The algorithm stops until the halting condition is reached, e.g. the number of iterations is equal to the block sparsity level or the residual is less than a threshold. After determining the support set, the sparse representation of the signal can be recovered according to the least square method. Step 4 assures that signal residuals are orthogonal with all the support block through recursion, which will ensure optimal representation and reduce the number of iterations. Benefit from that, the algorithm has faster convergence rate and more superior efficiency and accuracy in the process of searching for support blocks of signals.

Theorem 2 [30] Any block $K$-sparse vector $\boldsymbol{x}$ can be recovered from measurements $\boldsymbol{y}=\boldsymbol{\Theta} \boldsymbol{x}$ using BOMP if the block-coherence satisfies $K d<\left(\mu_{B}^{-1}+d\right) / 2$, where $\mu_{B}$ is the block-coherence defined as

$$
\mu_{B}=\max _{l . r \neq l} \frac{1}{d} \rho(M[l, r])
$$

where $M[l, r]=\boldsymbol{\Theta}^{\mathrm{H}}[l] \boldsymbol{\Theta}[r]$ is the $(l, r)$ th $d \times d$ block of the $N \times N$ matrix $M=\Theta^{\mathrm{H}} \Theta$. When $d=1, \mu_{B}$ decreases to the conventional definition of (4).
If $\boldsymbol{x}$ is treated as a conventional random $K d$-sparse vector without exploiting knowledge of the block-sparse structure, a sufficient condition for perfect recovery using OMP is $K d<\left(\mu^{-1}+d\right) / 2$. We know that the coherence is an equivalent condition of RIP and $\delta_{K \mid I} \leqslant \delta_{k}$ has been proved in Section 4.1.1. It is easy to deduce that $\mu_{B} \leqslant \mu$. Thus exploiting the block structure guarantees the recovery of a potentially higher sparsity level.

Considering the computational complexity, we suppose that the number of compressed sampling points is $M$, the number of Nyquist sampling points is $N$, the traditional sparsity level is $k$ and the block sparsity level is $K$. Then the computational complexity of OMP is $O(M \cdot N \cdot k)$, while the BOMP is $O(M \cdot N \cdot K)$. Obviously, the addition of block sparsity can significantly reduce the computational complexity.

The application range of BOMP is not limited to strictly uniform block sparse signals, and it has good performance in non-uniform block sparse signals and even compressible signals with certain clustering characteristics. Although the size of the block will affect the reconstruction performance, setting a smaller block size can have a certain degree of adaptability for different block sparse signals. Thus it can be effective to deal with practical problems.

\subsection{Simulation results and analysis}

In this section, an availability verification of the block greedy algorithm for the multi-band narrowband signal is given by simulation. We choose the typical ideal multiband narrowband signal as the original signal, which is expressed as follows:

$$
x(t)=\sum_{i=1}^{L} E_{i} \cdot \operatorname{sinc}\left(B\left(t-\tau_{i}\right)\right) \cos \left(2 \pi f_{i}\left(t-\tau_{i}\right)\right)
$$

where $L$ is the number of sub-bands, $B$ is the sub-band bandwidth, $E_{i}$ is the energy coefficient, and $f_{i}$ is the carrier frequency. The signal above can represent the ideal case of a radar reconnaissance receiver to receive unknown signals at a certain time.

In simulation, we set $L=4, B=2 \mathrm{MHz}, E_{i}=[5,8]$, $f_{i}=[15 \mathrm{MHz}, 35 \mathrm{MHz}]$. The simulation sampling rate is $f_{s}=100 \mathrm{MHz}$ and the signal length is $N=2048$. The original signal is shown in Fig. 5 and we can see that only a few frequency bands are occupied in a large bandwidth.

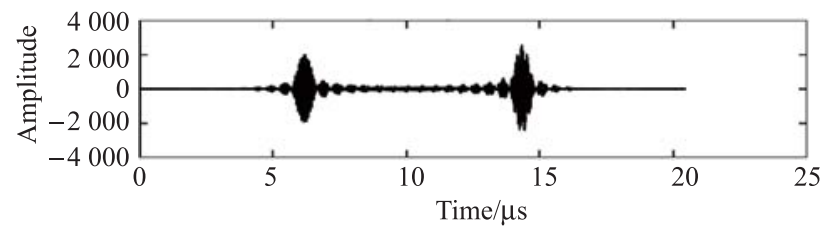

(a) Time domain 


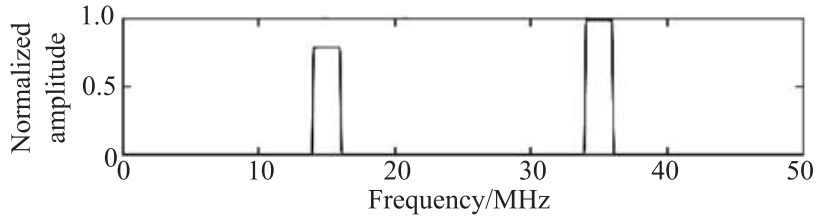

(b) Frequency domain

Fig. 5 Original signal

The sparsity level is $k=168$, the number of sample is $M=400$, the block length is $d=3$, the block sparsity level is $K=k / d=56$. When the iteration number of BOMP is 56 , a representative refactoring case is presented in Fig. 6:
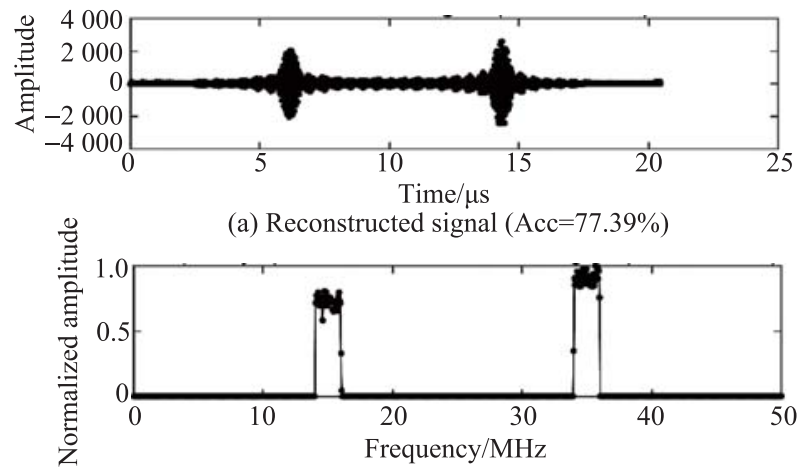

(b) Frequency spectrum of reconstructed signal (Acc=90.42\%)

Fig. 6 Reconstruction with 56 times iteration by BOMP

It can be seen that when the number of samples is $M=400$ and the sub-sampling rate is $Q=0.27$, the reconstructed accuracy is $90.42 \%$ in the frequency domain and $77.39 \%$ in the time domain. Most of the information of the original signal is reconstructed basically. In the same sub-sampling rate, OMP cannot reconstruct the original signal obviously. Simulation results under the same conditions by OMP are shown in Fig. 7.
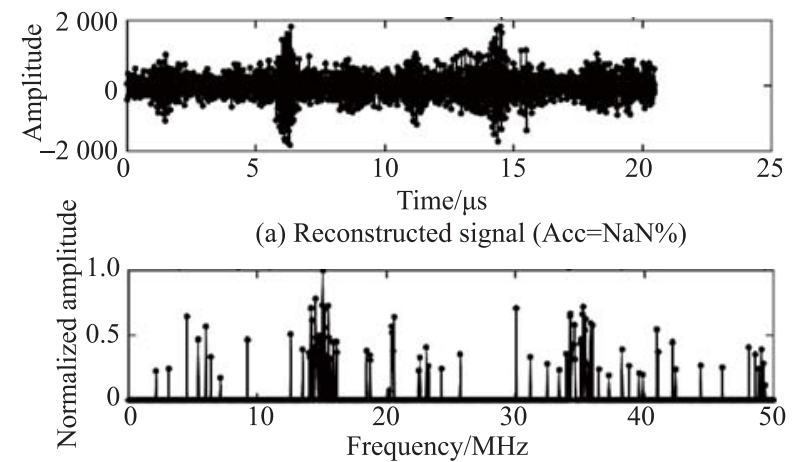

(b) Frequency spectrum of reconstructed signal ( $\mathrm{Acc}=28.24 \%$ )

Fig. 7 Reconstruction with 168 times iteration by OMP

It is noteworthy that BOMP inevitably leads to the decline of reconstruction accuracy because it selects the atom block by block rather than a single one in each iteration with the principle of maximum correlation, which will influence the process of computing the projected value of orthogonal basis in the pseudo-inverse operation. This problem can be compensated by increasing the number of iteration appropriately. In the conventional greedy algorithm, the number of iteration should be equal to the sparsity level which represents the number of all non-zero values narrowly. However, the narrowband radar signal is a compressible signal instead of a strict sparse signal. Hence, the concept of generalized sparsity can be exploited to the algorithm. The generalized sparse signal can use $K$ larger values to represent the original signal approximately in the sparsity domain while the remaining value is close to 0 but not equal to 0 exactly. It means that the sparsity level of a generalized sparse signal is relative. The greater the sparsity level is taken, the more information can be retained. In the contrary, the smaller the sparsity level is taken, the sparser and more anamorphic the signal is. Thus, increasing the number of iteration, which means increasing the generalized sparsity level $K$, can find more detailed information to improve the reconstruction accuracy. Thus, it is quite necessary to apply the generalized sparsity in engineering practice. Especially, when the receiver is faced with the noisy signal, the whole CS system will lose the prerequisite of sparsity if we only consider it in narrow sense. When the number of iteration increases to 112 , simulation results are shown in Fig. 8.
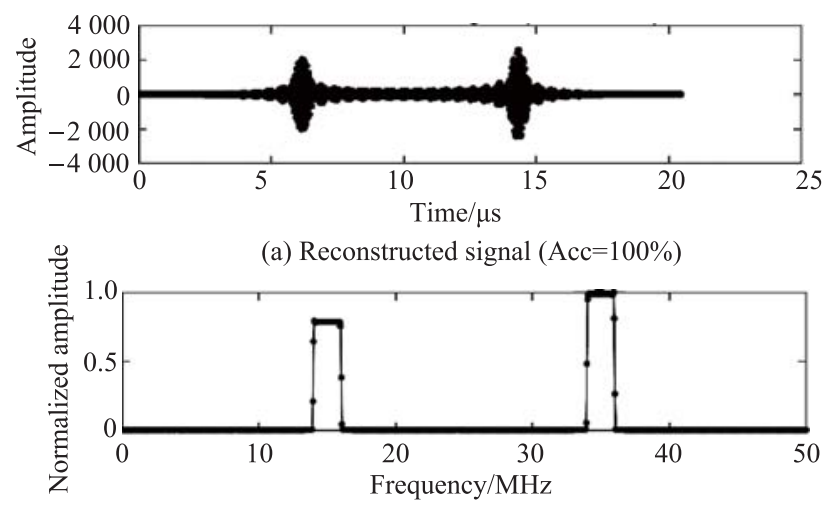

(b) Frequency spectrum of reconstructed signal $(\mathrm{Acc}=100 \%)$

Fig. 8 Reconstruction with 112 times iteration by BOMP

As can be seen from the simulation results, at the same sub-sampling rate, the accuracy of the reconstruction both in frequency domain and time domain rises to $100 \%$ after increasing the number of iteration. It can be proved that the multi-band narrowband signal can be reconstructed effectively and completely by BOMP under the AIC sampling model.

Fig. 9 depicts the relationship between sub-sampling 
rate and probability of reconstruction using OMP and BOMP. For BOMP, the block size is $d=3,21$, and set the iteration number twice the block sparsity level $K$ to ensure the reconstruction effect. For OMP, we set the iteration number $k$ and $2 k$ respectively, where $k$ is the traditional sparsity level of the original signal. Each case operates 500 times Monte Carlo simulation.

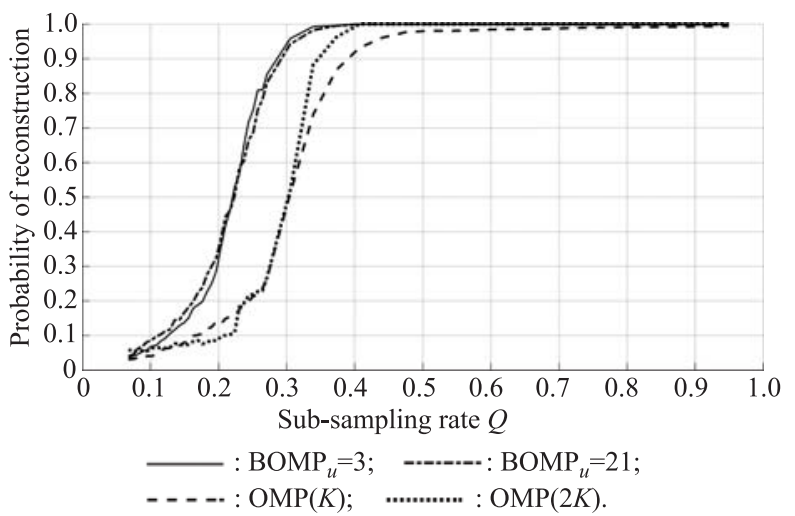

Fig. 9 Relationship between $Q$ and probability of reconstruction

The relationship between the reconstruction time and the number of sub-sample $M$ is shown in Fig. 10.

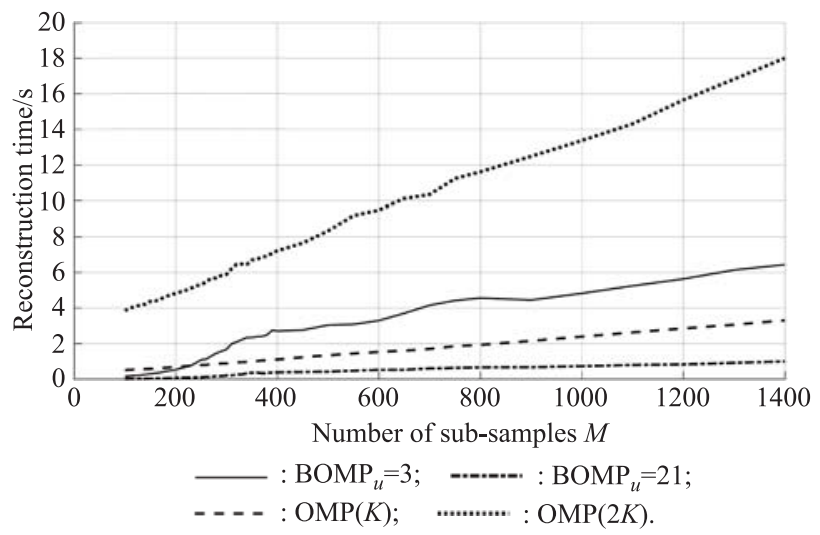

Fig. 10 Relationship between $M$ and reconstruction time

First, we analyze the difference of reconstruction results between OMP and BOMP. On one hand, the probabilities of refactoring have reached $100 \%$ while the sub-sampling rate is 0.4 except the situation of OMP with $k$ times iteration. We can see that the BOMP does better than OMP of double iteration obviously when the sub-sampling rate is less than 0.4. On the other hand, OMP of double iteration pays a huge cost of calculation and time to exchange the promotion of reconstruction probability as can be seen from Fig. 10. By contrast, BOMP can spend half the time of OMP or less to get the same reconstruction probability. Although OMP of $k$ times iteration is faster than BOMP in case of $d=3$, its reconstruction probability cannot reach
$100 \%$ even the sub-sampling rate is close to one. This result also confirms that signals with continuous spectrum cannot be effectively reconstructed, in other words, the traditional greedy algorithm under the AIC sampling model can only be applied to the limited information rate signal.

Next, we mainly compare reconstruction results of BOMP in different block length. Fig. 9 shows that when a relatively large block is used, the effect of reconstruction is better than the small block at a low sub-sampling rate range. Following with the rise of sub-sampling rate, the reconstruction probability of small block can catch up with and gradually surpass the large case. It can be well explained in the step of atomic search that the larger block can get more information when the sampling is inadequate, while the smaller block can provide higher accuracy in calculating least-square solutions. Considering the reconstruction time, the larger block has shorter time for block reconstruction.

Fig. 11 depicts the relationship between the sparsity level and the probability of reconstruction. We augment the signal sparsity level by increasing the number of subbands. The number of compression sampling $M$ is 400 . For BOMP, the block size is $d=2$. Both algorithms are carried out respectively with two different iterations and each case operates 500 times Monte Carlo simulations. The results show that the performance of $2 k$ iterations of OMP is better than that of BOMP in a small sparsity level with the increase of sub-bands. $K$ iterations of BOMP has a higher efficiency when the sparsity level is large than 100, however it is inferior in accuracy at the same time. Obviously, the $2 K$ iterations of BOMP has the best reconstruction performance and the appropriate computational complexity. Overall, the results of Fig. 11 felicitously correspond to the simulation conclusions of Fig. 9 and Fig. 10.

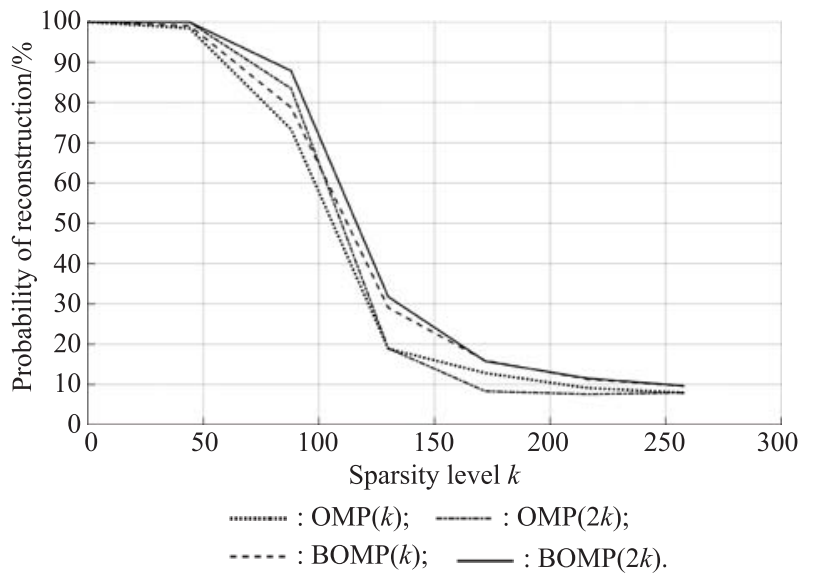

Fig. 11 Relationship between $k$ and probability of reconstruction

The two sets above of simulation results show that BOMP has noteworthy advantages in the AIC sub- 
sampling model for receiving the multi-narrowband signal. However there are also some problems need to be solved. We all know that the signal which the reconnaissance receiver faces is totally unknown, regardless of the block length and the block sparsity level. Therefore, in practice, a residual threshold or a preset block sparsity level can be set to adapt to the unknown block sparse level. At the same time, a smaller block length such as $l=2$ can be set to guarantee the versatility of the algorithm. In general, the adaptive problem of the block length and the sparsity level has yet to be resolved.

\section{Conclusions}

AIC is generally considered only to be used for the signal with the limiting information rate in CS. The reason is that the method of selecting the single atom form orthogonal bases in traditional greedy algorithms is not effective for non-strict sparse signals which have continuous spectrum. Combining the concept of block sparsity, this paper takes advantage of the potential relevance of support sets to improve the reconstruction performance of AIC for receiving the multi-band narrowband signal. Theoretical analyses prove that combining the concept of block sparsity with CS can increase the probability of reconstruction and reduce the sub-sampling rate. This paper also attempts to increase the number of iterations to improve the reconstruction accuracy. Simulation results show that the block greedy algorithm can complete reconstruction of the multi-band narrowband signal more effectively under the AIC sampling model. It has great potential to reduce the sampling rate of radar reconnaissance receiver, break the bottleneck of $\mathrm{ADC}$ and further meet the requirement of real-time characteristics.

\section{References}

[1] D. Donoho. Compressed sensing. IEEE Trans. on Information Theory, 2006, 52: 1289-1306.

[2] E. Candès, J. Romberg, T. Tao. Robust uncertainty principles: exact signal reconstruction from highly incomplete frequency information. IEEE Trans. on Information Theory, 2006, 52: $489-509$

[3] E. Candès, M. Wakin. An introduction to compressive sampling. IEEE Signal Processing Magazine, 2006, 52(2): $21-$ 30.

[4] J. A. Tropp, J. N. Laska, M. F. Duarte, et al. Beyond Nyquist: efficient sampling of sparse bandlimited signals. IEEE Trans. on Information Theory, 2010, 56(1): 520-544.

[5] M. Mishali, Y. C. Eldar. Blind multiband signal reconstruction: Compressed sensing for analog signals. IEEE Trans. on Signal Processing, 2009, 57(3): $993-1009$.

[6] Y. C. Eldar. Compressed sensing of analog signals in shiftinvariant spaces. IEEE Trans. on Signal Processing, 2009, 57(8): 2986-2997.

[7] M. Mishali, Y. C. Eldar. Spectrum-blind reconstruction of multi-band signals. Proc. of the IEEE International Conference on Acoustics, Speech and Signal Processing, 2008:
$3365-3368$

[8] J. N. Laska, S. Kirolos, Y. Massoud, et al. Random sampling for analog-to-information conversion of wideband signals. Proc. of the IEEE Dallas Circuits and Systems Workshop, 2006: $119-122$.

[9] S. Kirolos, T. Ragheb, J. N. Laska, et al. Practical issues in implementing analog-to-digital converters. Proc. of the International Workshop on System-on-Chip for Real-Time Applications, 2006: $141-146$.

[10] S. Kirolos, J. Laska, M. Wakin, et al. Analog-to-information conversion via random demodulation. Proc. of the IEEE Dallas Circuits and Systems Workshop, 2006.

[11] T. Ragheb, S. Kirolos, J. N. Laska, et al. Implementation models for analog-to-information conversion via random sampling. Proc. of the IEEE International Midwest Symposium on Circuits and Systems, DOI:10.1109/MWSCASA.2007. 4488599.

[12] J. N. Laska, S. Kirolos, M. F. Duarte, et al. Theory and implementation of an analog-to-information converter using random demodulation. Proc. of the IEEE Symposium on Circuits and Systems, 2007.

[13] S. Becker, J. Bobin, E. J. Candès. NESTA: a fast and accurate first-order method for sparse recovery. SIAM Journal on Imaging Sciences, 2011, 4 (1): 1-39.

[14] P. Daponte, L. De Vito, G. Iadarola, et al. Effects of PRBS jitter on random demodulation analog-to-information converters. Proc. of the IEEE Metrology for Aerospace, 2016: 630-635.

[15] M. A. Davenport, S. R. Schnelle, J. P. Slavinsky, et al. A wideband compressive radio receiver. Proc. of the Military Communications Conference, 2010: 1193-1198.

[16] J. Treichler, M. Davenport, J. Laska, et al. Dynamic range and compressive sensing acquisition receivers. Proc. of the 7th U.S./Australia Joint Workshop on Defense Applications of Signal Processing, 2011.

[17] J. Treichler, M. Davenport, R. Baraniuk. Application of compressive sensing to the design of wideband signal acquisition receivers. Proc. of the 6th U.S./Australia Joint Workshop on Defense Applications of Signal Processing, 2009.

[18] Y. Lu, W. Guo, X. Wang, et al. Distributed streaming compressive spectrum sensing for wide-band cognitive radio networks. Proc. of the IEEE 73rd Vehicular Technology Conference, 2011: $1-5$.

[19] R. D'Angelo, M. Trakimas, S. Aeron, et al. Experimental results on wideband spectrum sensing using random sampling ADC in 90nm CMOS. Proc. of the IEEE International Symposium on Circuits and Systems, 2013: 1970 - 1973.

[20] M. Mishali, Y. C. Eldar, J. A. Tropp. Efficient sampling and stable reconstruction of wide band sparse analog signals. Proc. of the IEEE 25th Convention of Electrical and Electronics Engineers, 2008: 290-294.

[21] M. Mishali, A. Elron, Y. C. Eldar. Sub-Nyquist processing with the modulated wideband converter. Proc. of the IEEE International Conference on Acoustics, Speech and Signal Processing, 2010: 3626-3629.

[22] M. Mishali, Y. C. Eldar. From theory to practice: sub-nyquist sampling of sparse wideband analog signals. IEEE Journal of Selected Topics on Signal Processing, 2010, 4(2): 375 - 391.

[23] D. L. Donoho. For most large underdetermined systems of linear equations, the minimal L1 norm solution is also the sparsest solution. Communications on Pure and Applied Mathematics, 2006, 59(6): $797-829$.

[24] S. Boyd, L. Vandenberghe. Convex optimization. Cambridge, UK: Cambridge University Press, 2004.

[25] E. Candès, T. Tao. Decoding by linear programming information theory. IEEE Trans. on Information Theory, 2005, 51(12): $4203-4215$. 
[26] J. A. Tropp. Algorithms for simultaneous sparse approximation. Part II: convex relaxation. signal process. Sparse Approximations in Signal and Image Processing, 2006, 86: 589-602.

[27] J. A. Tropp. Algorithms for simultaneous sparse approximation. Part I: greedy pursuit. signal process. Sparse Approximations in Signal and Image Processing, 2006, 86: 572-588.

[28] N. Lee. MAP support detection for greedy sparse signal recovery algorithms in compressive sensing. IEEE Trans. on Signal Processing, 2016, 64(19): 4987 - 4999.

[29] Y. C. Eldar, H. Bolcskei. Block-sparsity: coherence and efficient recovery. Proc. of the IEEE International Conference on Acoustics, Speech, and Signal Processing, 2009: 2885-2888.

[30] Y. C. Eldar, P. Kuppinger, H. Bolcskei. Compressed sensing of block-sparse signal: uncertainly relations and efficient recovery. IEEE Trans. on Signal Processing, 2011, 58(6): $3042-$ 3054.

[31] M. Shekaramiz, T. K. Moon, J. H. Gunther. On the blocksparsity of multiple-measurement vectors. Proc. of the IEEE Signal Processing and Signal Processing Education Workshop, 2015: 220-225.

[32] S. Shah, T. Goldstein, C. Studer. Estimating sparse signals with smooth support via convex programming and block sparsity. Proc. of the IEEE Conference on Computer Vision and Pattern Recognition, 2016: 5906-5915.

[33] R. G. Baraniuk, E. Candes, M. Elad, et al. Applications of sparse representation and compressive sensing. Proceedings of the IEEE, 2010, 98(6): $906-909$.

[34] Y. C. Eldar, M. Mishali. Block-sparsity and sampling over a union of subspaces. Proc. of the 16th International Conference Digital Signal Processing, 2009: 1-8.

[35] B. Thomas, E. Mike. Sampling theorems for signals from the union of finite-dimensional linear subspaces. IEEE Trans. on Information Theory, 2009, 55(4): $1872-1882$.

\section{Biographies}

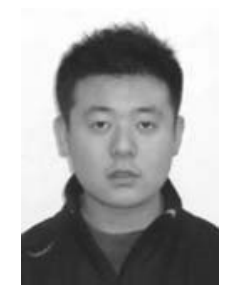

Hongyi Xu was born in 1992. He received his B.S. degree in electronic and information engineering, from Harbin Engineering University, Harbin, China, in 2014. He is currently working towards his M.S. and Ph.D. degrees in information and communication engineering at Harbin Engineering University, Harbin, China. His research interests include radar signal processing and compressive sensing. He is currently participating in academic exchange in Beijing Institute of Technology.

E-mail: xhyxwxag@126.com

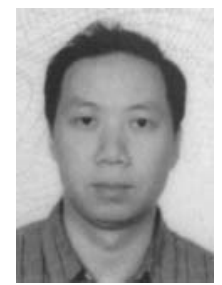

Haiqing Jiang was born in 1981. He received his B.S. and Ph.D. degrees, both in signal and information processing, from Beijing Institute of Technology, Beijing, China, in 2003 and 2008, respectively. $\mathrm{He}$ is currently working as a lecturer in School of Information and Electronics at Beijing Institute of Technology. His research interests include radar signal processing and digital receiver technology. E-mail: haiqingd99@bit.edu.cn

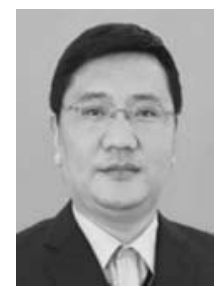

Chaozhu Zhang was born in 1970. He received his B.S. degree in electronics and information engineering from Harbin Institute of Technology in 1993, M.S. degree in communications and information systems and Ph.D. degree in signal and information processing from Harbin Engineering University in 2002 and 2006 respectively. He is a professor for Harbin Engineering University, China. He is a member of IEEE, academician of the Chinese Aerospace Society and the Heilongjiang Biomedical Engineering Society. His researches include signal processing applications in radar and communications and image processing.

E-mail: zhangchaozhu@hrbeu.edu.cn 